\title{
Pooled sputum to optimise the efficiency and utility of rapid, point-of-care molecular SARS-CoV-2 testing
}

\author{
Alison Burdett ${ }^{1{ }^{*}}$, Christofer Toumazou ${ }^{1,2 \dagger}$, Rashmita Sahoo ${ }^{1}$, Adam Mujan ${ }^{1}$, Tsz-Kin Hon ${ }^{1}$, Judith Bedzo-Nutakor ${ }^{1}$, \\ Nicola Casali', Maria Karvela', Mohammadreza Sohbati', Graham S. Cooke ${ }^{3,4}$, Gary W. Davies ${ }^{5 \dagger}$ and \\ Luke S. P. Moore ${ }^{3,4,5^{*+}}$ (DD
}

\begin{abstract}
Background: As SARS-CoV-2 testing expands, particularly to widespread asymptomatic testing, high sensitivity point-of-care PCR platforms may optimise potential benefits from pooling multiple patients' samples.

Method: We tested patients and asymptomatic citizens for SARS-CoV-2, exploring the efficiency and utility of CovidNudge (i) for detection in individuals' sputum (compared to nasopharyngeal swabs), (ii) for detection in pooled sputum samples, and (iii) by modelling roll out scenarios for pooled sputum testing.

Results: Across 295 paired samples, we find no difference $(p=0.1236)$ in signal strength for sputum (mean amplified replicates (MAR) 25.2, standard deviation (SD) 14.2, range 0-60) compared to nasopharyngeal swabs (MAR 27.8, SD 12.4, range 6-56). At 10-sample pool size we find some drop in absolute strength of signal (individual sputum MAR 42.1, SD 11.8, range 13-60 vs. pooled sputum MAR 25.3, SD 14.6, range 1-54; $p<0.0001$ ), but only marginal drop in sensitivity (51/53,96\%). We determine a limit of detection of $250 \mathrm{copies} / \mathrm{ml}$ for an individual test, rising only four-fold to 1000 copies/ml for a 10-sample pool. We find optimal pooled testing efficiency to be a 12-31-sample model, yet as prevalence increases, pool size should decrease; at 5\% prevalence to maintain a 75\% probability of negative first test, 5-sample pools are optimal.
\end{abstract}

Conclusion: We describe for the first time the use of sequentially dipped sputum samples for rapid pooled point of care SARS-CoV-2 PCR testing. The potential to screen asymptomatic cohorts rapidly, at the point-of-care, with PCR, offers the potential to quickly identify and isolate positive individuals within a population "bubble".

Keywords: COVID-19, Coronavirus, Molecular diagnostics, PCR

\footnotetext{
*Correspondence: alison.burdett@dnanudge.com; luke.moore@nhs.net

${ }^{+}$Alison Burdett and Alison Burdett are Joint first authors; contributed equally

${ }^{\dagger}$ Gary W. Davies and Luke S. P. Moore Joint senior authors; contributed

equally

'DnaNudge Ltd, Imperial College White City Campus, The Translation and

Innovation Hub, Level 11, 84 Wood Lane, London W12 OBZ, UK

${ }^{3}$ North West London Pathology, Imperial College Healthcare NHS Trust,

Fulham Palace Road, London W6 8RF, UK

Full list of author information is available at the end of the article
}

(C) The Author(s). 2021 Open Access This article is licensed under a Creative Commons Attribution 4.0 International License, which permits use, sharing, adaptation, distribution and reproduction in any medium or format, as long as you give appropriate credit to the original author(s) and the source, provide a link to the Creative Commons licence, and indicate if changes were made. The images or other third party material in this article are included in the article's Creative Commons licence, unless indicated otherwise in a credit line to the material. If material is not included in the article's Creative Commons licence and your intended use is not permitted by statutory regulation or exceeds the permitted use, you will need to obtain permission directly from the copyright holder. To view a copy of this licence, visit http://creativecommons.org/licenses/by/4.0/. The Creative Commons Public Domain Dedication waiver (http://creativecommons.org/publicdomain/zero/1.0/) applies to the data made available in this article, unless otherwise stated in a credit line to the data. 


\section{Background}

Molecular testing, predominantly reverse transcriptase polymerase chain reaction (RT-PCR), remains the standard of care for detection of SARS-CoV-2 due to high sensitivity and specificity. However laboratory-based RTPCR requires significant infrastructure, is often centralised, and turnaround times frequently exceed $24 \mathrm{~h}$. This has driven supplementary testing with rapid lateral flow antigen tests, but these often exhibit sub-optimal sensitivity $[1,2]$. Developing molecular diagnostic tests for use outside of laboratory settings, with retained 'gold standard' test performance characteristics, could accelerate clinical decision making, enabling effective triage and infection control measures in frontline clinical and community settings.

The CovidNudge assay potentially meets this need, as a point-of-care, real-time RT-PCR test without the need for significant laboratory facilities or sample preparation, with a turnaround time of $90 \mathrm{~min}$. The platform comprises a single-use DnaCartridge and a processing unit (the NudgeBox) with six viral targets $(R d R p-I P 2, R d R p$ $I P 4, e$-gene, $n 1, n 2, n 3)$ and one host gene as a sample adequacy control (Ribonuclease P, RNaseP) (supplementary Fig. 1a, supplementary Table 1), generating positive, negative, or indeterminate results (supplementary Table 2) [3]. The sensitivity of this assay compared with laboratory-based PCR using nasopharyngeal swab samples was found to be $97 \%$ (95\% CI 89.6-99.6) with a specificity of $100 \%$ (95\% CI 98.5-100) [3].

As the need for COVID-19 testing continues to develop, particularly for asymptomatic screening, nearpatient rapid testing must further respond. Currently each NudgeBox processes one sample at a time, and although an increase in throughput can be accommodated by deploying additional platforms, the use of pooled patient samples may enable incremental throughput [4]. The USA Food and Drug Administration (FDA) currently recommends two approaches to specimen pooling [5]; pooled aliquots of transport media each containing a single patient sample (sample pooling), or combined swabs from multiple patients into a single volume of transport media (swab pooling). UK guidelines currently only recommend sample pooling, as swab pooling provides no mechanism to deconvolve individual positive samples [6]. So far, pooling has not been widely used in clinical or community settings. For laboratory-based molecular platforms this is likely because of; a lack of validated pooling techniques; delayed reporting of positive pools from subsequent individual sample retesting; and difficulties in fine resolution identification of appropriate low-prevalence cohorts to deploy in.

For point-of-care RT-PCR platforms, potential benefits from pooling may be more easily realised. Near-patient tests enable a positive pool to be quickly followed up with individual testing, while a negative pool enables rapid de-escalation of restrictions. However, neither of the pooling methods described earlier are compatible with the CovidNudge platform, as the system has no requirement for liquid transport media, therefore no simple method exists for combining swabs from individual patients. As an alternative, sputum can be directly expectorated without the use of a swab, with comparable sensitivity and specificity to oro- or nasopharyngeal samples in previous analyses [7]. Although sputum is considered a difficult sample to process with traditional RTPCR platforms as the high viscosity poses challenges in transfer with a fixed volume transfer bulb, these limitations do not occur on the CovidNudge platform since a sample can be directly transferred to the DnaCartridge using an appropriate swab.

This study explores the efficiency and utility of CovidNudge among symptomatic [8] patients and asymptomatic citizens (i) for detection of SARS-CoV-2 in individuals' sputum (in comparison to nasopharyngeal swabs), (ii) for detection of SARS-CoV-2 in pooled sputum, and (iii) by modelling roll out scenarios for pooled sputum testing.

\section{Method}

\section{Sputum sampling}

To investigate whether sputum samples are compatible with the CovidNudge platform, we undertook a comparative analysis of nasopharyngeal swab samples with sputum. Testing took place in September and October 2020 using samples from two separate groups: patients admitted to hospital via the emergency department at Chelsea \& Westminster NHS Foundation Trust, and asymptomatic members of the London Symphony Orchestra. Testing of emergency admissions at Chelsea \& Westminster NHS Foundation Trust was done as a service evaluation approved by the Trust COVID-19 Testing Committee. All participants consented to supplying a contemporaneous nasopharyngeal swab and sputum sample (supplementary method).

Sputum samples were collected into a sample tube with inactivating agent (Oragene500, DNAgenotek) [9]. Nasopharyngeal samples were tested on the CovidNudge platform; from hospitalised patients at the point of care as part of normal clinical practice; from asymptomatic members of the London Symphony Orchestra at the point of collection (Covent Garden, London). Sputum samples from both hospitalised patients and orchestra members were tested in laboratory facilities at DnaNudge premises (Imperial College Translation and Innovation Hub, Wood Lane, London). All sputum 
samples were opened, sampled, and inserted into the DnaCartridge within a biosafety cabinet (NUARE: Class II 12469:2000, Model No: NU-543-300S) following Health and Safety Executive (HSE) requirements relating to handling of samples from persons with respiratory illnesses. To test the sputum samples, an RNA/DNA buccal swab (SK-2, Isohelix) was used, rubbed in the inactivated sputum for 5 seconds, then inserted into the DnaNudge cartridge (supplementary Fig. 1b). The cartridge was then inserted into the NudgeBox and a test run following standard procedure [3].

To determine whether the number of replicates amplifying during the CovidNudge test could serve as a semi-quantitative marker for viral load, for the positive samples we correlated the number of replicates amplifying on the CovidNudge platform against cycle threshold values obtained using laboratory PCR platforms (supplementary method).

\section{Sputum pooling}

Pools were tested with one positive sample and the rest as negative samples, using a single buccal swab to sequentially "dip" the sputum samples. Initial exploratory analysis was performed starting at a pool of two and then incrementing the pool by adding further negative samples each time, up to a maximum pool size of 40 and 30. In practice these pool sizes are unlikely to be practical unless prevalence is very low, therefore the characteristics of pooling were further elucidated using a pool size $n=10$. UK guidelines currently recommend a pool size between 6 and 12 [6]. USA FDA guidelines regarding the validation of $n$-pooled tests recommend that samples from at least 20 positive patients and $(20 \times \mathrm{n})$ negative patients should be collected and tested with one positive and $(n-1)$ negative samples per pool [5]. For our validation we took a larger number of positive samples $(n=51)$ to allow us to vary the position of the positive sample within the pool during the test (i.e. positive sample in first position, second position,... ninth position, tenth position) at least 3 times. The pooled samples were dipped in turn with a single Isohelix swab (supplementary method), then inserted into a DnaCartridge and tested following standard procedure [3]. Negative samples dipped subsequent to the positive patient sample were discarded as contaminated; negative samples dipped prior to the positive sample were retained for reuse. Positive samples were resampled a maximum of three times, as each sampling introduced dilution from addition of negative sputum into the positive sample.

\section{Limits of detection for pooling}

A negative sputum sample was spiked with viral genetic material (Microbiologics HE0062S process control pellet,
Lot: HE0062-01, Expiry: 2022-01-31) dissolved in molecular water. The viral solution was serially diluted and aliquots of $25 \mathrm{uL}$ were added to a $25 \mathrm{uL}$ sputum sample. These sequentially diluted samples were then absorbed onto an Isohelix swab and run on the CovidNudge platform using established methods [3] to determine the limit of detection (LOD).

\section{Optimising pooling size}

The efficiency gained through pooling of samples is highly dependent on the prevalence of positive patients in the cohort to be tested. We modelled pooling efficiency as a function of prevalence, considering two scenarios:

- Single pooled test: if the pooled test is reported as positive, the patient samples are all individually tested to determine which of the samples is positive

- Nested pooled test: if the pooled test is positive, smaller patient pools are repeated to narrow down the search for the positive result(s).

To explore the relative efficiency of single versus nested pooling, we simulated the result of different pooling scenarios (supplementary method). We selected a range of single and nested pooling scenarios, with a maximum initial pool size of 12 as per UK guidelines [6]. We also limited the total number of nested testing cycles to three (i.e. one or two rounds of pooling with a final individual testing round) to minimise delays to results which would detract from the benefit of having a rapid RT-PCR test at the point of care. For each initial pool size, all possible input sample permutations were evaluated. For a pool size of $n$, this results in $2^{n}$ input sample vectors. Each input vector was evaluated in turn to determine the total number of tests that would need to be run in the given pooling scenario. Finally, the probability of the specific input vector being realised was calculated as a function of prevalence.

\section{Statistical analysis}

Descriptive statistics were used, with chi squared tests for categorical variables, t-test for normally distributed continuous data (normal distribution was determined using the Kolmogorov-Smirnov test) and Mann-Whitney $\mathrm{U}$ test for non-parametric data. Pearson's correlation was used as a measure of strength of association when comparing sample type and semi-quantification.

\section{Results}

\section{Sputum sampling}

Paired nasopharyngeal and sputum samples were obtained from symptomatic [8] and asymptomatic hospital patients admitted through the emergency department 
diagnosed as SARS-CoV-2 positive $(n=74)$ or negative $(n=103)$, and asymptomatic screening from members of the London Symphony Orchestra $(n=118)$. 295 paired samples were obtained.

First, for the 74 positive patient samples we determined whether the number of replicates amplifying during the CovidNudge test can serve as a semi-quantitative marker for viral load when compared to cycle threshold values from laboratory RT-PCR platforms (Pearson's correlation coefficient, $\mathrm{r}=-0.71, p<0.001$, Fig. 1A). Second, comparing this semi-quantitative measure of positivity between paired nasopharyngeal and sputum samples, we find them comparable (Table 1, Fig. 1B). The mean number of replicates amplifying for sputum
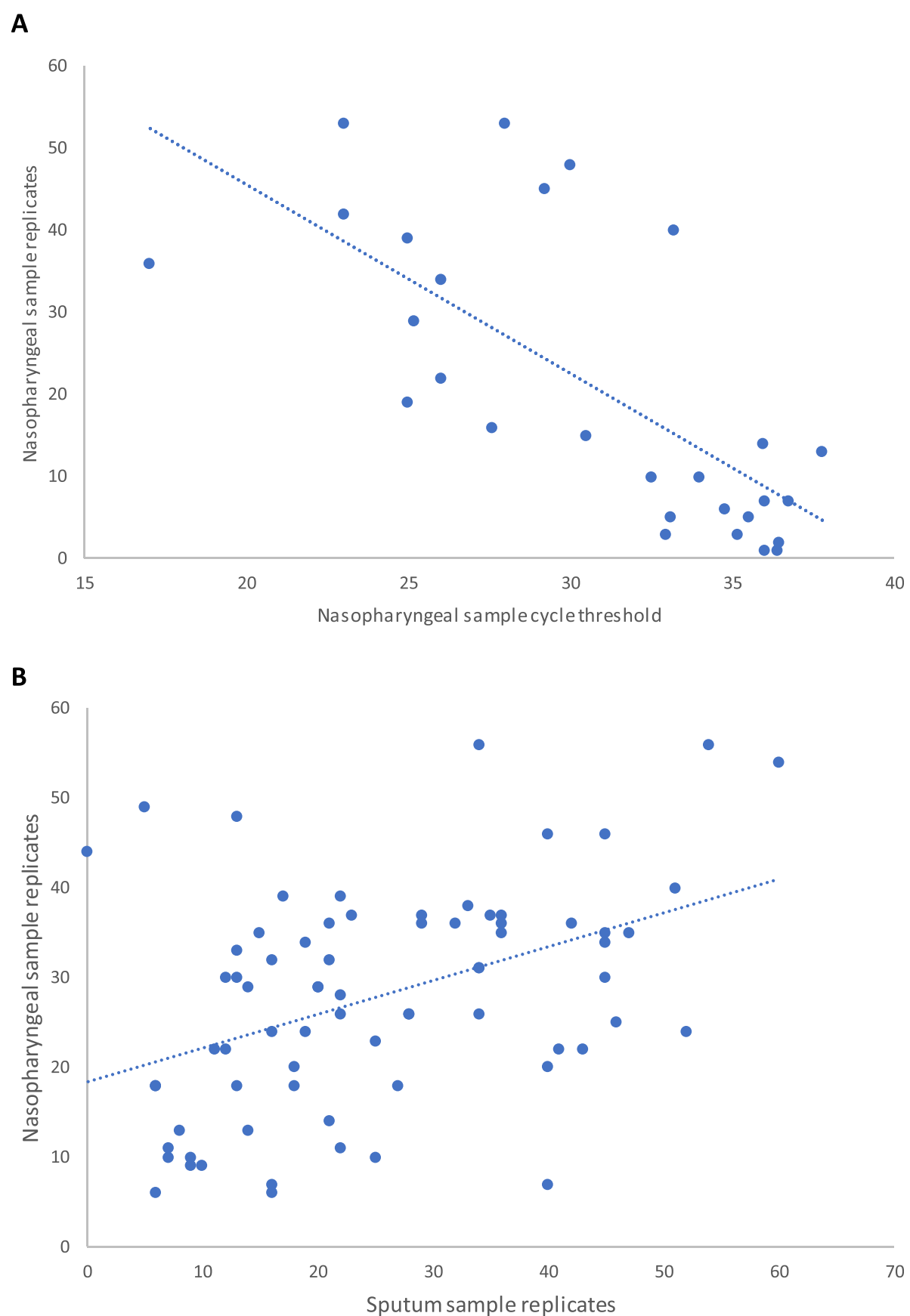

Fig. 1 Comparison of CovidNudge amplified replicates (1A) versus laboratory RT-PCR cycle threshold, and (1B) for nasopharyngeal swabs versus sputum samples 
Table 1 Nasopharyngeal and sputum paired samples tested on the CovidNudge platform, London, 2020

\begin{tabular}{llll}
\hline & & \multicolumn{2}{l}{ SPUTUM SAMPLES } \\
\cline { 3 - 4 } & & POSITIVE & NEGATIVE \\
\hline NASOPHARYNGEAL & POSITIVE & 73 & 1 \\
SAMPLES & NEGATIVE & 0 & 221 \\
\hline
\end{tabular}

Legend: Sputum samples demonstrated $98.65 \%$ sensitivity $(95 \% \mathrm{Cl}=92.7-$ 99.97\%) and $100 \%$ specificity $(95 \% \mathrm{Cl}=98.3-100 \%)$ against nasopharyngeal samples

was 25.2 (standard deviation (SD) 14.2, range 0-60) and for nasopharyngeal was 27.8 (SD 12.4, range 6-56). Both sets of data were shown to be normally distributed (sputum samples: $\mathrm{D}=0.126, p=0.183$; nasopharyngeal samples: $\mathrm{D}=0.065, p=0.899)$ and not significantly different $-2.6(95 \% \mathrm{CI}=-5.93$ to $0.73, p=0.124)$.

Three outlying results were identified with high number of nasopharyngeal replicates but low replicates in sputum samples (Fig. 1B). Two results had at least some detectable sputum replicates (nasopharyngeal 49, sputum 5, and nasopharyngeal 48, sputum 13), these two patients had 4 days of illness prior to swabbing; one had well controlled diabetes mellitus and the other was at the extreme of old age. One result had no detectable sputum replicates (nasopharyngeal 44, sputum 0), this patient was day 14 of illness and had been admitted for a non-COVID related illness and had no respiratory symptoms at the time of illness.

\section{Sputum pooling}

At the extreme end of the iterative pool-size ranging exercise, the platform was able to report a positive result from a single positive sputum sample in a pool of 40 and was repeatable in $3 / 5$ pools $(3 / 5$ detected $>13$ replicates, $2 / 5$ detected only 2 replicates and were therefore defined as indeterminate).

To evaluate the more functional 10-pool testing, 53 pools were run with a single positive and 9 negative samples (example run in Fig. 2A-F) to determine sensitivity, and 200 negative samples in all-negative pools to determine specificity (Table 2 ). The mean replicates amplifying for individual sputum samples was 42.1 (SD 11.8, range 13-60) and for pooled sputum samples was 25.3 (SD 14.6, range 1-54). Both sets of data were shown to be normally distributed (single sputum samples: $\mathrm{D}=0.168, p=0.090$; pooled sputum samples: $\mathrm{D}=0.122, p=0.381$ ), with a significant difference between single and pooled sputum replicates 16.8 (95\% $\mathrm{CI}=11.7$ to $21.9, p<0.0001$ ).

The 'position' of the positive sample in the pool (from first to tenth) was evaluated, with the positive sample placed in each position among the pool sampling 3 times (Fig. 2G). This demonstrated no significant correlation $(\mathrm{r}=0.06, p=0.68)$ between sample position and number of replicates.

\section{Limits of detection for pooling}

For individual sample testing the LOD was measured as 250 copies per swab; this climbed to 1000 copies per swab for 10 pool testing. The 4-fold increase in LOD for a 10-pool test suggests dipping a swab into successive sputum samples provides less sample dilution than the expected $\mathrm{n}$-fold reduction in viral concentration for an n-pool test.

\section{Putting pooling into practice}

For the scenarios simulated, all pooling strategies have an efficiency (i.e. ratio of number of tests required without pooling, compared to mean number of tests required with pooling) greater than 1 for prevalence up to $10 \%$ (Fig. 3A \& B). In all cases simulated, the nested pooling scenario results in a higher efficiency than the simple pooling case; however the trade-off is a longer wait for the final confirmatory result in case of a positive result in one or more of the pooled samples.

\section{Discussion}

The CovidNudge system provides rapid near-patient diagnostic capability for SARS-CoV-2 testing. To expand the utility of this platform, we find high levels of concordance in test performance characteristics between nasopharyngeal sampling and sputum. Equally importantly we find pooling of sputum for testing on the CovidNudge platform has no drop in sensitivity, and only marginal fall in the limits of detection. These two aspects together offer the opportunity to optimise the efficiency of novel diagnostic pathways for both patients and for citizens who may need to work or mix in close proximity.

While pooling of samples has the potential to offer significant efficiency gains in terms of consumables, the balance of time-to-result needs to be carefully considered for each potential use-setting. There are now examples of pooling in emerging COVID-19 outbreak scenarios $[10,11]$ and pooling of sputum for surveillance has already been advocated [12], and initial evaluations undertaken [13]. However for direct clinical care it is imperative to know the definitive result for a patient as soon as possible, and preferably at the point of care (e.g. when planning for an emergency surgery or to optimise utilisation of side room capacity) [14]. In such settings, the requirement for immediacy of results may rule out the use of laboratory based pooling because of the risk 

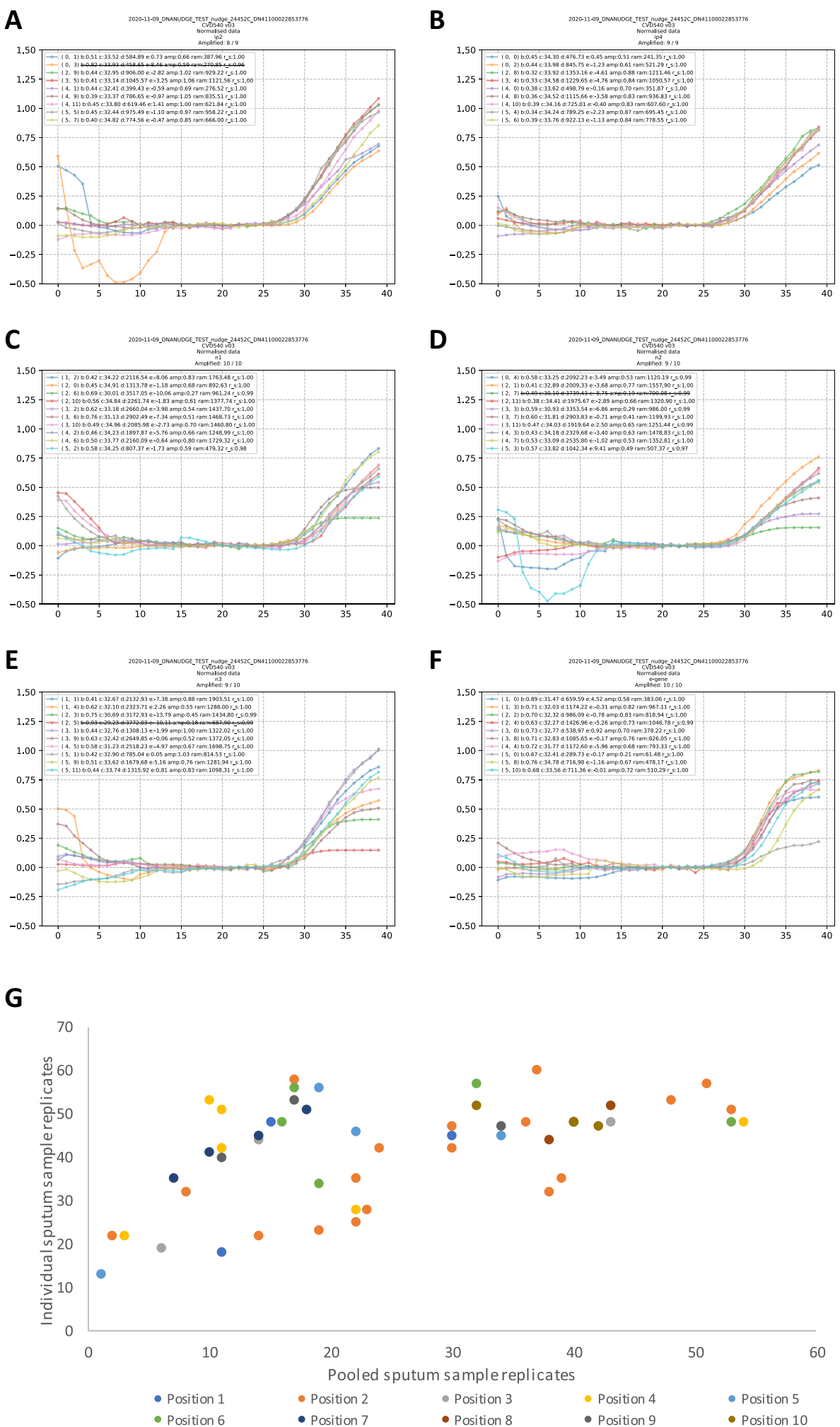

Fig. $\mathbf{2}$ CovidNudge PCR amplification curves for a pool of 1 positive and 9 negative samples across all six gene targets $(2 \mathbf{A}=R d R p-I P 2,2 \mathbf{B}=$ RdRp-IP4, 2C=n1, 2 $\mathbf{D}=n 2,2 \mathbf{E}=n 3,2 \mathbf{F}=$ e-gene), and with variation of the position of the positive across the 10-pool (2G). Legend: $2 \mathbf{A}-\mathbf{F}$. X-axis PCR cycle, Y-axis replicate well threshold. All 6 viral target genes showed amplification in most replicates. $2 \mathbf{G}$. Coloured dots denote how testing the positive sputum sample in a pool of 10 varied when the positive sample altered position in relation to the negatives (from position 1-10) 
Table 2 Test performance characteristics for pooled sputum samples on the CovidNudge platform, London, 2020

\begin{tabular}{lllll}
\hline & & POOLED SAMPLES & & \\
\cline { 3 - 5 } & & POSITIVE & INDETERMINATE & NEGATIVE \\
\hline INDIVIDUAL & POSITIVE & 51 & 2 & 0 \\
SAMPLES & INDETERMINATE & 0 & 0 & 0 \\
& NEGATIVE & 0 & 0 & 200 \\
\hline
\end{tabular}

Legend: 53 patients with positive individual samples were tested with 200 negative results. Pools were either tested as 10 negative samples, or one positive sample with 9 negative samples. An indeterminate result is reported when only one or two replicates amplify, i.e. the signal is at the limits of detection. Pooled samples demonstrated $96.23 \%$ sensitivity $(95 \% \mathrm{Cl}=87.0-99.5)$ and $100 \%$ specificity $(95 \% \mathrm{Cl}=98.2-100)$ against individual samples

that a second round of testing may be required. However there are specific use cases where near-patient pooling (such as we present here) is desirable, particularly when testing asymptomatic population groups. Practical use cases include regular screening of population cohorts ("bubbles") that are in regular and close contact either inside or outside of normal household groups such as; care home staff, residents, and visitors; family and support networks; workplaces, theatres and concerts, sports teams; school and university classroom groups; healthcare workers. Other researchers have identified the efficiency benefits of sample pooling at the point of care particularly in environments where test resources are scarce $[15,16]$, and the method described here warrants further investigation to ascertain utility in these settings.

Our sputum sample validation has demonstrated for the first time that a single inactivated sputum sample can be dipped multiple times, without requirements for pipetting small volumes needed in other evaluations of pooling $[10,11,13,17]$, which is impractical at the point of care. Our single pool-dipped swab can then be inserted directly into the DnaCartridge for processing due to the unique swab-to-result nature of the platform. Before n-pool testing, each sputum sample can be individually dipped with a swab and these swabs stored for later individual testing, should the pool test return a positive result.

To ensure accurate resulting for pooled samples, in the current DnaNudge platform, a pooled sample is registered using the multiple patient identifiers of each patient in the pool. Following a pooled negative result, an individual negative result is generated for each patient for onwards reporting into hospital records. A pooled positive result does not generate a result for all patients but instead flags the requirement for immediate retesting of each individual. This avoids patients who may be negative within a positive pool being erroneously reported for public health follow-up.

Our study has several limitations. First, although the validation of sputum samples against nasopharyngeal samples included both symptomatic and asymptomatic patients, in asymptomatic patients the terminology "sputum" may be somewhat misleading since the samples are unlikely to come from the lower respiratory tract. However, in all but one of the cases, the oral (salivary) sample provided did produce a positive result for SARS-CoV-2 for patients who had tested positive through nasopharyngeal sampling, as found elsewhere [18]. Second, whilst we correlated the number of positive CovidNudge replicates to laboratory-based PCR cycle threshold as a semi-quantitative measure, this is not a true representation of viral load, and this could impact our measurement of pooling to be able to detect lowpositive patients in a large pool. To mitigate this we did undertake formal limit of detection analysis, as noted, and our finding of a four-fold fall in LOD in a 10 pool, while not large, must be considered in the context of how pooling is being deployed. Third, while singlepatient use of the CovidNudge is a low-skilled activity, enabling use of the platform in a variety of near-patient and community scenarios, the multi-stepped process of pooling would require training, and dedicated cohorts of trained personnel should be used to support this aspect of its use. Finally, it is important to be cognizant with all COVID-19 diagnostics of the need to consider (and test for) variants of concern, and potentially other coronaviridae [19], and the CovidNudge gene targets will be continually evaluated for their utility and changed where needed.

\section{Conclusions}

We describe for the first time the use of sequentially dipped sputum samples using a commercially available buccal swab for rapid near-patient pooled SARS-CoV-2 RT-PCR testing. The sequential dipping of sputum minimises loss of sensitivity through dilution, while also retaining individual samples should a positive test result require further individual testing. The potential to screen asymptomatic cohorts rapidly, at the point of care, with the superior test performance characteristics of RT-PCR (compared to lateral flow devices) $[1,2]$ offers the potential to quickly identify and isolate positive individuals within a population "bubble", including in family units, workplaces, and social gatherings. 
A

Pool and nested pool efficiency comparison

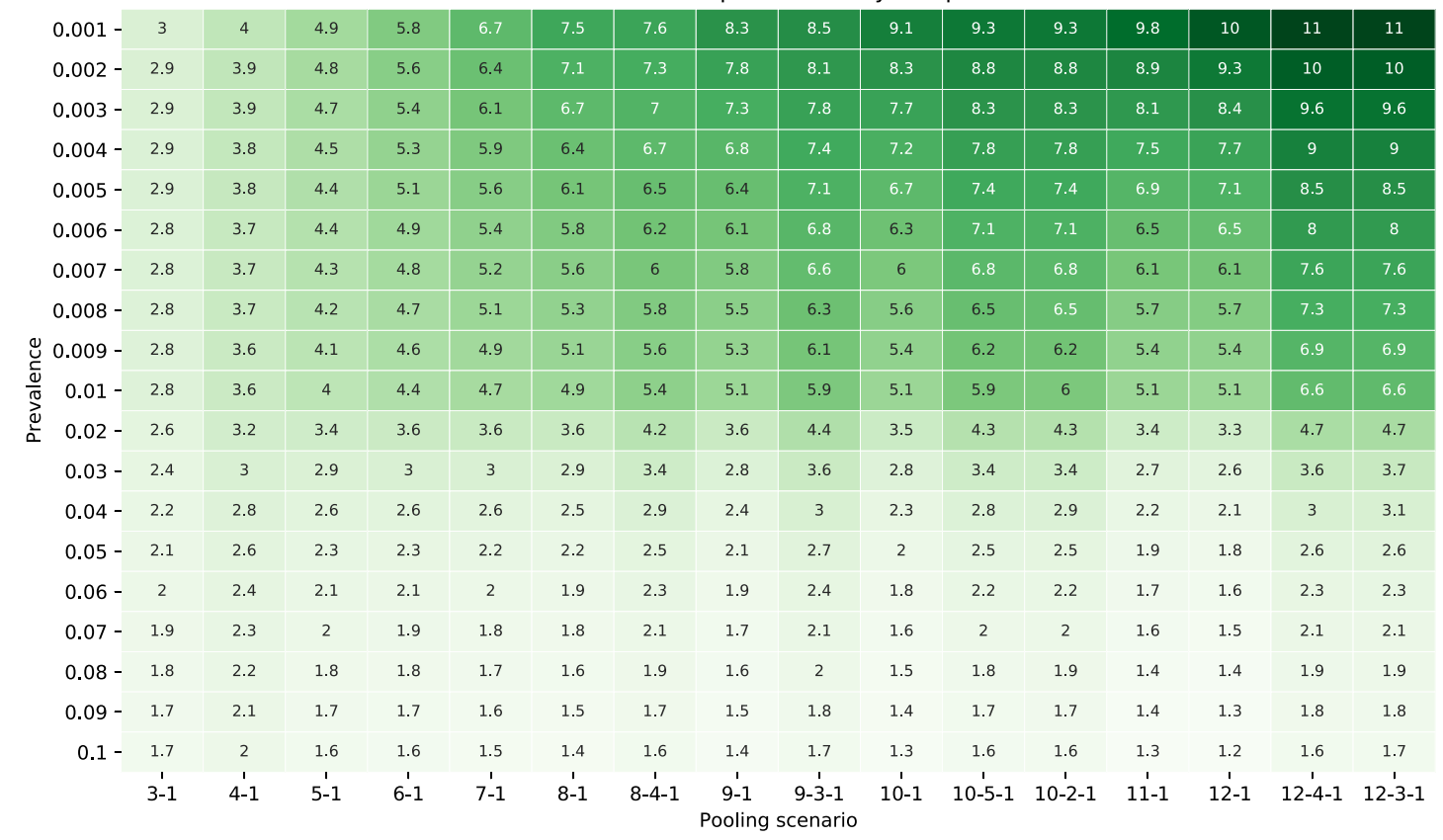

B

Probability of first test negative per pool scenario for prevalences of $0.1 \%-10 \%$

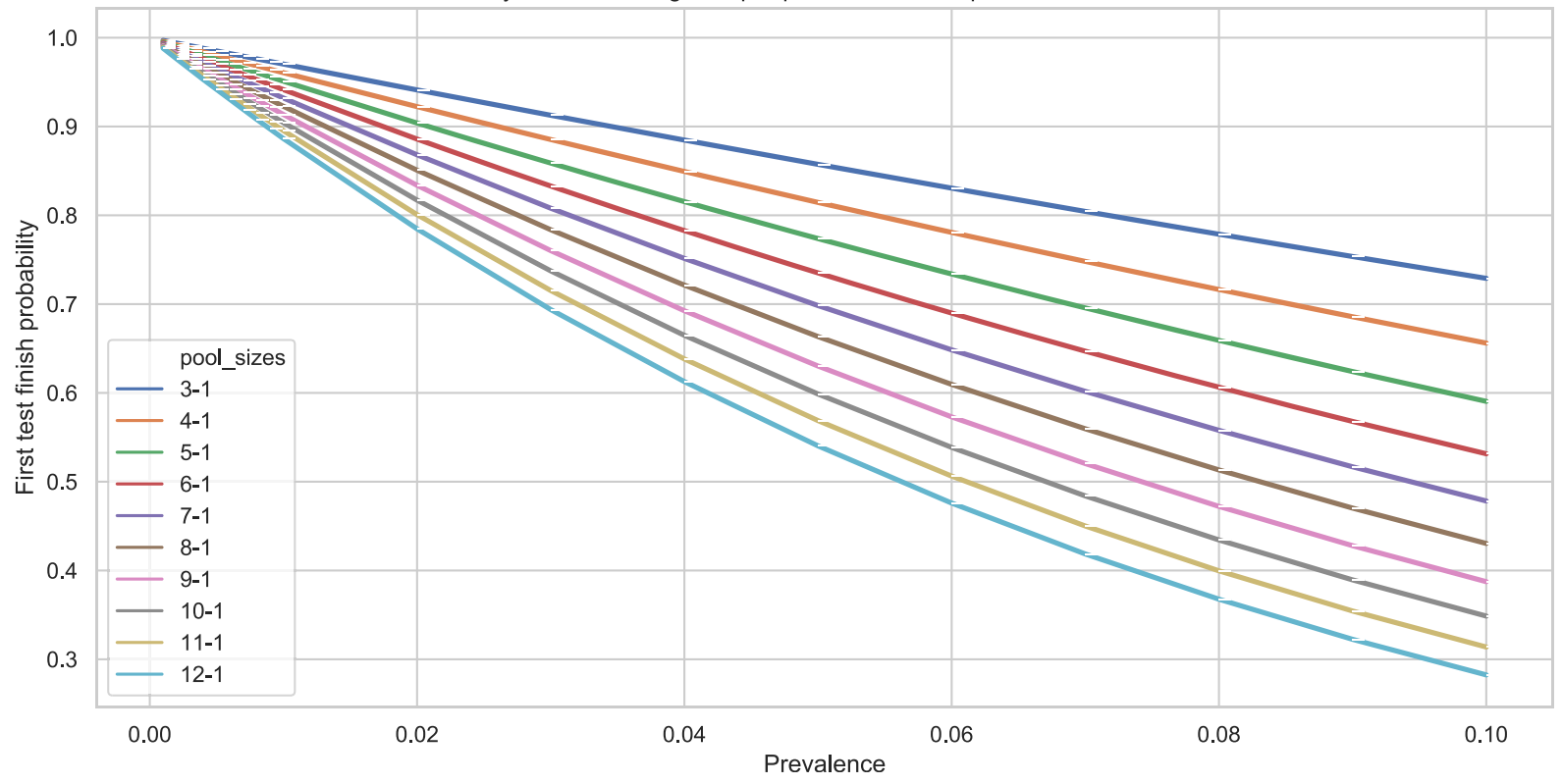

Fig. 3 Relative testing efficiency for various pool-size strategies for CovidNudge sputum PCR testing (3A) across climbing COVID-19 prevalence with (3B) probability of a negative first pool. Legend: $3 \mathbf{A}$. Calculation of the relative efficiency for different pooling strategies as a function of prevalence, where relative efficiency $(E r)$ is defined as $\mathrm{Er}=\frac{\text { Number tests required without pooling }}{\text { Average number of test required with pooling }} 3 \mathbf{B}$. For prevalence below $2 \%$, pooling of up to 12 samples has a high probability (> 75\%) of returning a negative result of first test. As prevalence increases, to maintain high efficiency of pooling, the pool size should decrease. At $5 \%$ prevalence, to maintain a 75\% probability of a negative first test, the pool size should decrease to 5 


\section{Abbreviations}

CE mark: Conformité Européene mark; COVID-19: Coronavirus disease 2019; DNA: Deoxyribonucleic acid; Er: Relative efficiency; FDA: Food and drug administration; HSE: Health and safety executive; LOD: Limit of detection; RdRp: RNA-dependent RNA polymerase; RT-PCR: Reverse transcriptase polymerase chain reaction; RNA: Ribonucleic acid; SARS-CoV-2: Severe acute respiratory syndrome coronavirus 2; SD: Standard deviation; UK: United Kingdom; USA: United States of America

\section{Supplementary Information}

The online version contains supplementary material available at https://doi. org/10.1186/s12879-021-06316-z.

Additional file 1.

\section{Acknowledgements}

DnaNudge supplied the test cartridges and NudgeBox processing units. We acknowledge the contribution of the study participants and the staff of Imperial College National Health Service (NHS) Trust (London, UK), Chelsea and Westminster Hospital NHS Foundation Trust (London, UK), North West London Pathology, and the Imperial College Molecular Diagnostic Unit. LSPM acknowledges support from the National Institute of Health Research Imperial Biomedical Research Centre and the National Institute for Health Research Health Protection Research Unit in Healthcare Associated Infection and Antimicrobial Resistance at Imperial College London in partnership with Public Health England. The views expressed in this publication are those of the authors and not necessarily those of the NHS, the National Institute for Health Research, or the UK Department of Health.

\section{Authors' contributions}

Assay design and development was done by $\mathrm{CT}$ (genetics and bioengineering design), RS, NC (assay development and molecular biology), MS, T-KH (platform technology and cartridge design), MK (genetics and microbiology). Clinical evaluation was led by AM, JB-N, GD, AB, GSC, RS, and LSPM. Analysis was done by $A B$ and MS. The first draft of manuscript was written by $A B, C T$, and LSPM. All authors reviewed and approved the final manuscript.

\section{Funding}

Funding for consumables and research staff time was provided by DnaNudge.

\section{Availability of data and materials}

The datasets analysed during the current are available from the lead author (AB alison.burdett@dnanudge.com) on reasonable request, as long as this meets local ethics and research governance criteria.

\section{Declarations}

\section{Ethics approval and consent to participate}

This study was approved by the Chelsea \& Westminster NHS Foundation Trust COVID testing committee. The study was reviewed by the Chelsea \& Westminster NHS Foundation Trust Research and Development Office and deemed a verification of a CE marked in vitro diagnostic test, therefore the need individual informed consent was waived. Aggregated data was analysed in accordance to the UK Secretary of State for Health and Social Care general notice that under the Health Service Control of Patient Information Regulations (2002) patient data for a COVID-19 purposes may be used for research. The study was conducted in accordance with relevant guidelines and regulations including the Declaration of Helsinki.

\section{Consent for publication}

All authors consent to publication of this manuscript in this journal. This work has not been previously published in any other journal.

\section{Competing interests}

$A B, C T, R S, N C, A M, M S, M K, T-K H$ and JB are employees of DnaNudge. CT is the co-inventor of the DnaNudge CovidNudge system and is named on the patent for the method and apparatus for analysing biological specimens on the DnaNudge platform (US Patent No: US 10093 965.B2). LSPM has consulted for and/or received speaker fees from bioMerieux (2013-2021), Pfizer (2018-2021), Eumedica (2016-2021), Umovis Lab (2020-2021), Shionogi (2021), Pulmocide (2021), DNAelectronics (2015-18), and Dairy Crest (20172018), received research grants from the National Institute for Health Research (2013-2019), and CW+ Charity (2018-2021). GC is an NIHR research professor and investigator within the NIHR London in-vitro diagnostic cooperative. All other authors declare no competing interests.

\section{Author details}

${ }^{1}$ DnaNudge Ltd, Imperial College White City Campus, The Translation and Innovation Hub, Level 11, 84 Wood Lane, London W12 OBZ, UK. ${ }^{2}$ Department of Electrical and Electronic Engineering, Imperial College London, Exhibition Road, London SW7 2AZ, UK. ${ }^{3}$ North West London Pathology, Imperial College Healthcare NHS Trust, Fulham Palace Road, London W6 8RF, UK. ${ }^{4} \mathrm{NIHR}$ Health Protection Research Unit in Healthcare Associated Infections \& Antimicrobial Resistance, Imperial College London, Du Cane Road, London W12 0NN, UK. ${ }^{5}$ Chelsea and Westminster NHS Foundation Trust, 369 Fulham Road, London SW10 9NH, UK.

Received: 22 February 2021 Accepted: 14 June 2021

Published online: 08 July 2021

\section{References}

1. Fitzpatrick MC, Pandey A, Wells CR, Sah P, Galvani AP. Buyer beware: inflated claims of sensitivity for rapid COVID-19 tests. Lancet. 2021;397(10268):24-5. https://doi.org/10.1016/S0140-6736(20)32635-0.

2. Public Health England. Preliminary report from the Joint PHE Porton Down and University of Oxford SARS-CoV-2 test development and validation cell: rapid evaluation of lateral flow viral antigen detection devices (LFDs) for mass community testing. Public Health England; 2021. Available from: www. ox.ac.uk/sites/files/oxford/media_wysiwyg/UK\%20evaluation_PHE\%2 OPorton\%20Down\%20\%20University\%20of\%200xford_final.pdf. Accessed 23 Dec 2020.

3. Gibani MM, Toumazou C, Sohbati M, Sahoo R, Icely C, Karvela M, et al. Assessing a novel, lab-free, point-of-care test for SARS-CoV-2 (CovidNudge): a diagnostic accuracy study. Lancet Microbe. 2020;1(7):300-7.

4. Lohse S, Pfuhl T, Berkó-Göttel B, Rissland J, Geißler T, Gärtner B, et al. Pooling of samples for testing for SARS-CoV-2 in asymptomatic people. Lancet Infect Dis. 2020;20(11):1231-2. https://doi.org/10.1016/S1473-3099(20)30362-5.

5. United States of America Food and Drug Administration. Molecular Diagnostic Template for Commercial Manufacturers. FDA; 2020. Available from: https://www.fda.gov/medical-devices/coronavirus-disease-2019covid-19-emergency-use-authorizations-medical-devices/vitro-diagnosticseuas. Accessed 23 Dec 2020.

6. Public Health England. Pooling of asymptomatic SARS COV-2 COVID-19 samples for (PCR/or other) testing. Public Health England; 2020. Available from: https://www.england.nhs.uk/coronavirus/publication/pooling-of-a symptomatic-sars-cov-2-covid-19-samples-for-pcr-or-other-testing/. Accessed 23 Dec 2020.

7. Mohammadi A, Esmaeilzadeh E, Li Y, Bosch RJ, Li JZ. SARS-CoV-2 detection in different respiratory sites: a systematic review and meta-analysis. EBioMedicine. 2020:59:102903. https://doi.org/10.1016/j.ebiom.2020.102903.

8. Public Health England. COVID-19: investigation and initial clinical management of possible cases. Public Health England; 2020. Available from: https://www.gov.uk/government/publications/wuhan-novel-corona virus-initial-investigation-of-possible-cases/investigation-and-initial-clinicalmanagement-of-possible-cases-of-wuhan-novel-coronavirus-wn-covinfection. Accessed 23 Dec 2020.

9. DNAgenotek. Inactivation of SARS-CoV-2 in samples using Oragene, ORAcollect, and OMNIgene products from DNAgenotek. DNAgenotek; 2020 Available from: https://www.dnagenotek.com/US/pdf/MK-01430.pdf. Accessed 23 Dec 2020.

10. Singh AK, Nema RK, Joshi A, Shankar P, Nema S, Raghuwanshi A, et al. Evaluation of pooled sample analysis strategy in expediting case detection in areas with emerging outbreaks of COVID-19: a pilot study. PLoS One. 2020;15(9):e0239492. https://doi.org/10.1371/journal.pone.0239492.

11. Cabrera Alvargonzalez JJ, Rey Cao S, Pérez Castro S, Martinez Lamas L, Cores Calvo O, Torres Piñon J, et al. Pooling for SARS-CoV-2 control in care institutions. BMC Infect Dis. 2020;20(1):745. https://doi.org/10.1186/s12879020-05446-0. 
12. Fogarty A, Joseph A, Shaw D. Pooled saliva samples for COVID-19 surveillance programme. Lancet Resp Med. 2020;8(11):1078-80. https://doi. org/10.1016/S2213-2600(20)30444-6.

13. Mahmoud SA, lbrahim E, Thakre B, Teddy JG, Raheja P, Ganesan S, et al. Evaluation of pooling of samples for testing SARS-CoV- 2 for mass screening of COVID-19. BMC Infect Dis. 2021;21(1):360. https://doi.org/10.1186/s12879-021-06061-3.

14. Moore LSP. Near-patient SARS-CoV-2 molecular platforms: new-old tools for new-old problems. Lancet Resp Med. 2020;8(12):1161-3. https://doi.org/10.1 016/S2213-2600(20)30451-3.

15. Hogan CA, Sahoo MK, Pinsky BA. Sample pooling as a strategy to detect community transmission of SARS-CoV-2. JAMA. 2020;323(19):1967-9. https:// doi.org/10.1001/jama.2020.5445.

16. Becker MG, Taylor T, Kiazyk S, Cabiles DR, Meyers AF, Sandstrom PA Recommendations for sample pooling on the Cepheid GeneXpert ${ }^{\oplus}$ system using the Cepheid Xpert ${ }^{\oplus}$ Xpress SARS-CoV-2 assay. PLoS One. 2020;15(11): e0241959. https://doi.org/10.1371/journal.pone.0241959.

17. Chen F, Geng Z, Wang J, Liuchang W, Huang D, Xu Y, et al. Comparing two sample pooling strategies for SARS-CoV-2 RNA detection for efficient screening of COVID-19. J Med Virol. 2021;93(5):2805-9. https://doi.org/10.1002/jmv.26632.

18. Skolimowska K, Rayment M, Jones R, Madona P, Moore LSP, Randell P. Noninvasive saliva specimens for the diagnosis of COVID-19: caution in mild outpatient cohorts with low prevalence. Clin Microbiol Infect. 2020;26(12): 1711-3. https://doi.org/10.1016/j.cmi.2020.07.015

19. Pham VH, Gargiulo Isacco C, KCD N, Le SH TDK, Nguyen QV, Pham HT, et al. Rapid and sensitive diagnostic procedure for multiple detection of pandemic Coronaviridae family members SARS-CoV-2, SARS-CoV, MERS-CoV and $\mathrm{HCOV}$ : a translational research and cooperation between the Phan Chau Trinh University in Vietnam and University of Bari "Aldo Moro" in Italy. Eur Rev Med Pharmacol Sci. 2020;24(12):7173-91.

\section{Publisher's Note}

Springer Nature remains neutral with regard to jurisdictional claims in published maps and institutional affiliations.

Ready to submit your research? Choose BMC and benefit from:

- fast, convenient online submission

- thorough peer review by experienced researchers in your field

- rapid publication on acceptance

- support for research data, including large and complex data types

- gold Open Access which fosters wider collaboration and increased citations

- maximum visibility for your research: over $100 \mathrm{M}$ website views per year

At $\mathrm{BMC}$, research is always in progress.

Learn more biomedcentral.com/submissions 